Supporting Information for

\title{
Spectroscopic Study of Ensemble and Individual Graphene Quantum Dots
}

Yanyu Lei ${ }^{a}$, Jiayi Wang ${ }^{b}$, Pan Chen ${ }^{b}$, Daocheng Hong ${ }^{a}$, Sushu Wan ${ }^{a}$, Xiaoyong Deng ${ }^{*}, b$, Yuxi $\operatorname{Tian}^{*}, a$

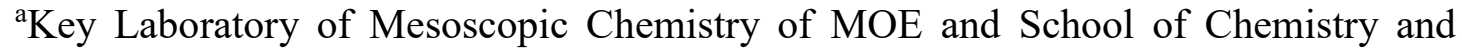
Chemical Engineering, Nanjing University, 210023, Nanjing, China

${ }^{\mathrm{b}}$ Institute of Nanochemistry and Nanobiology and School of Environmental and Chemical Engineering

Shanghai University, 200444, Shanghai, China

*Corresponding author.

E-mail address: xydeng@shu.edu.cn; tyx@nju.edu.cn 


\section{HRTEM images of GQDs and IGQDs}

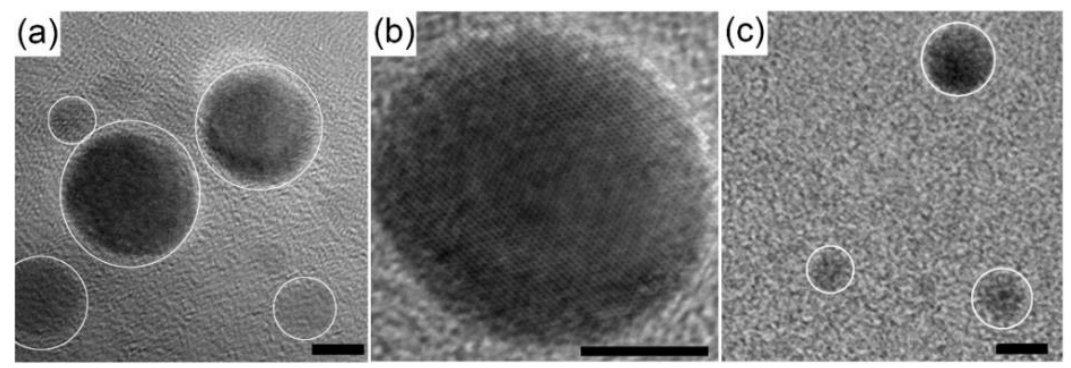

Figure S1 HRTEM images of GQDs ( $a$ and $b$ ) and IGQDs (c). (The scale is $5 \mathrm{~nm}$ )

\section{Size distribution of GQDs and IGQDs}
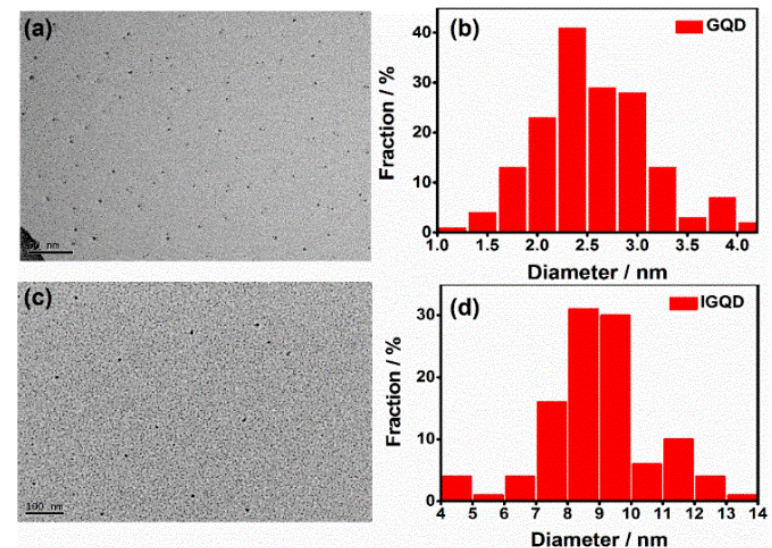

Figure S2 TEM images and corresponding diameter distributions of the GQDs $(a, b)$ and IGQDs (c, d), respectively. (The scale is $100 \mathrm{~nm}$ )

\section{XRD patterns of GQDs and IGQDs}

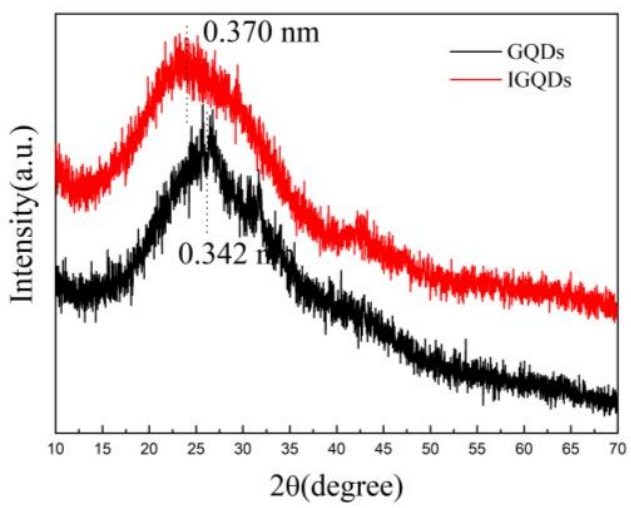

Figure S3 XRD patterns of GQDs (a) and IGQDs (b). 


\section{XPS spectra of GQDs and IGQDs}
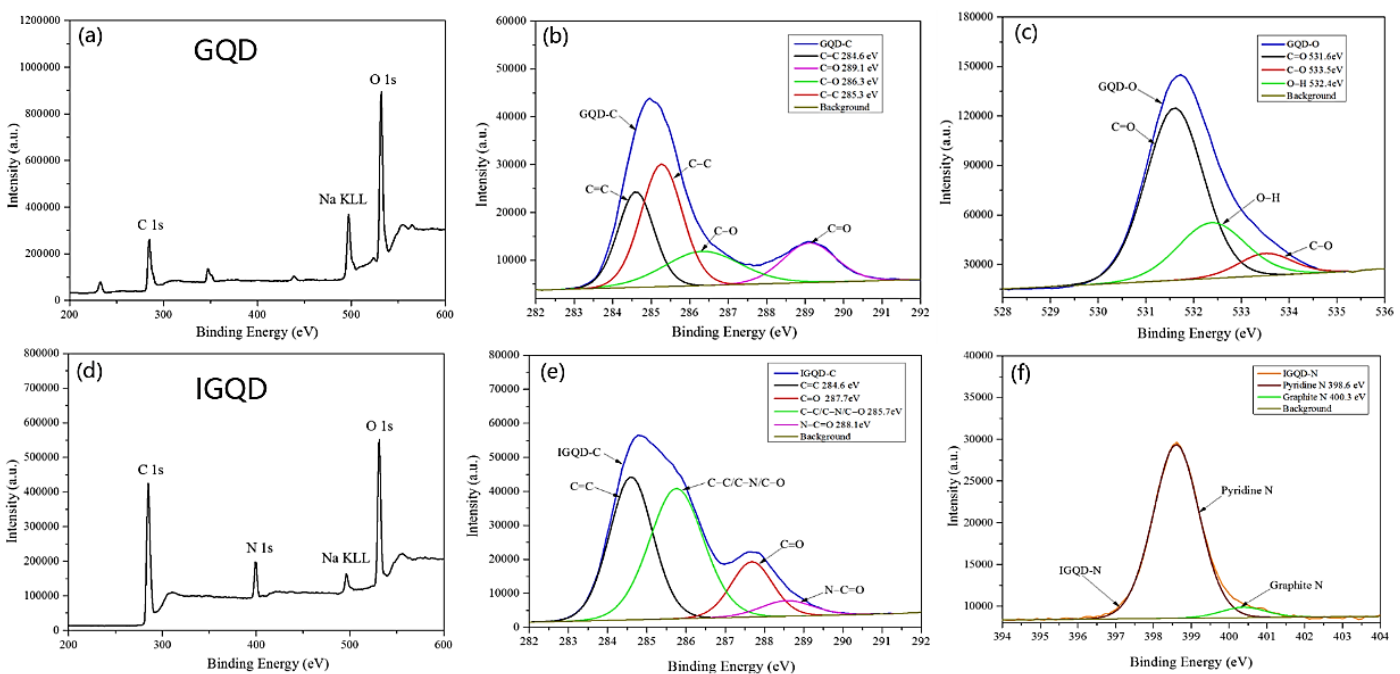

Figure S4 The full XPS spectra of GQDs (a) and IGQDs (d), with high-resolution C1s spectra of GQDs (b) and IGQDs (e), while high-resolution O1s spectrum of GQDs is shown in (c) and high-resolution N1s spectrum of IGQDs is shown in (f).

\section{PLE spectra of GQDs}

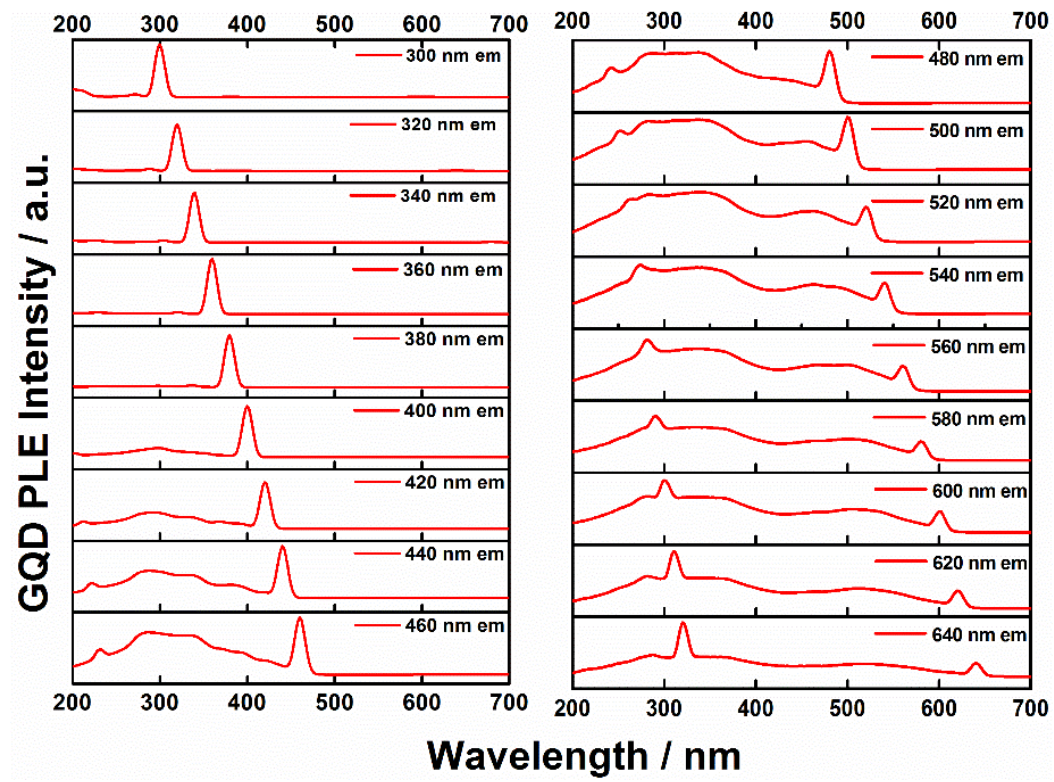

Figure S5 PL excitation spectra of GQDs (in $20 \mathrm{~nm}$ increments). 
6. PLE spectra of IGQDs

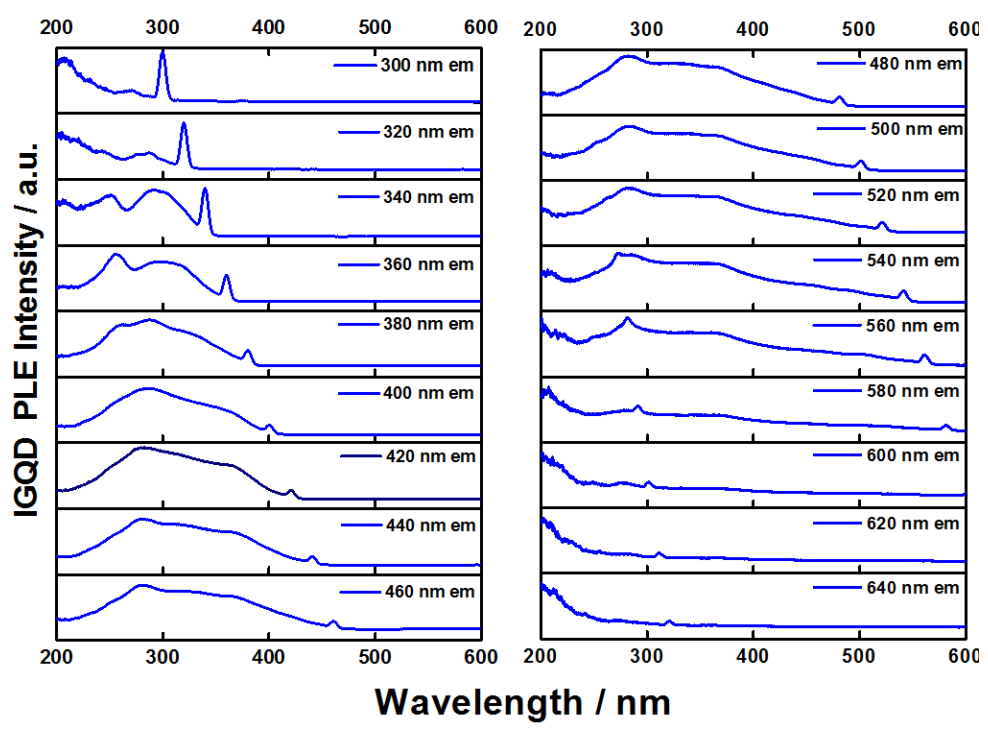

Figure S6 PL excitation spectra of IGQDs (in $20 \mathrm{~nm}$ increments).

7. Typical luminescence intensity traces of individual GQDs/IGQDs
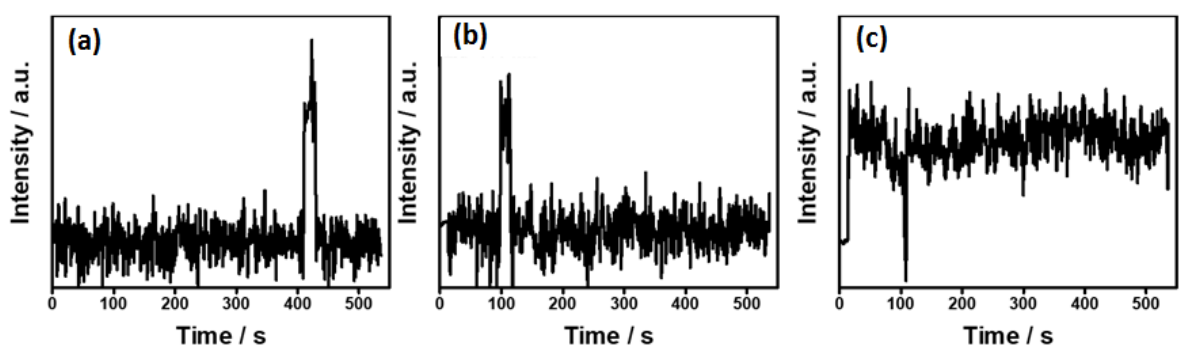

Figure S7 typical luminescence intensity trances of individual GQD (a) and $\operatorname{IGQD}(b, c)$.

8. The wavelength distribution and PL spectra for GQDs with different excitation wavelengths 
(a)

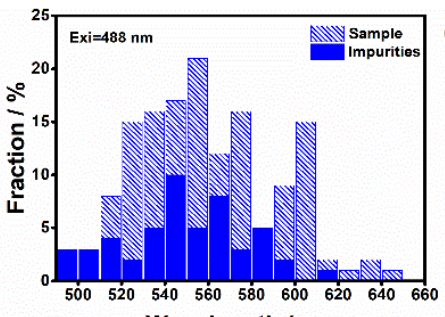

(b)

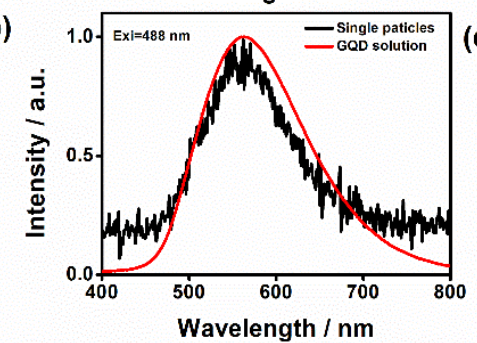

(c)

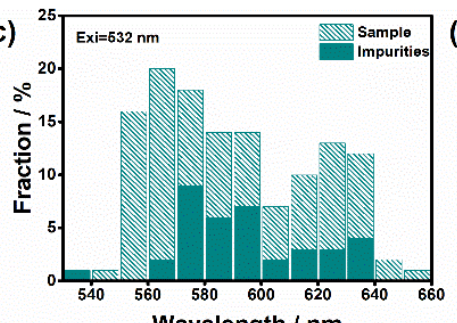

Wavelength $/ \mathrm{nm}$

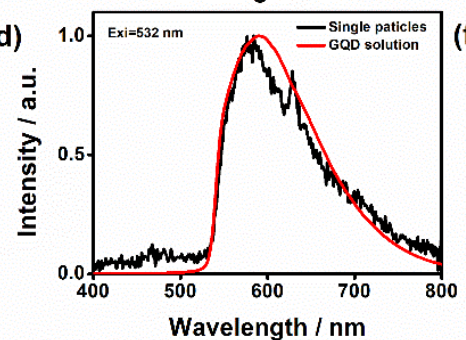

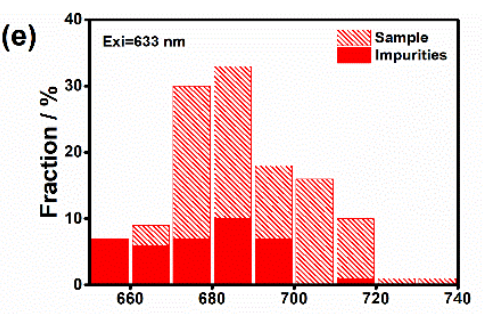

Wavelength / nm

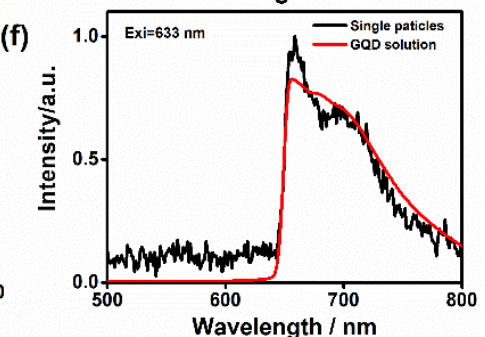

Figure S8 Histogram of peak position of PL spectra from individual GQDs (a, c, e), the sum of all the PL spectra from individual dots (black lines in $b, d, f$ ), and the spectra of GQDs solutions (red lines in b, d, f) when excited with $488 \mathrm{~nm}(\mathrm{a}, \mathrm{b}), 532$ $\mathrm{nm}(\mathrm{c}, \mathrm{d})$ and $633 \mathrm{~nm}(\mathrm{e}, \mathrm{f})$. 